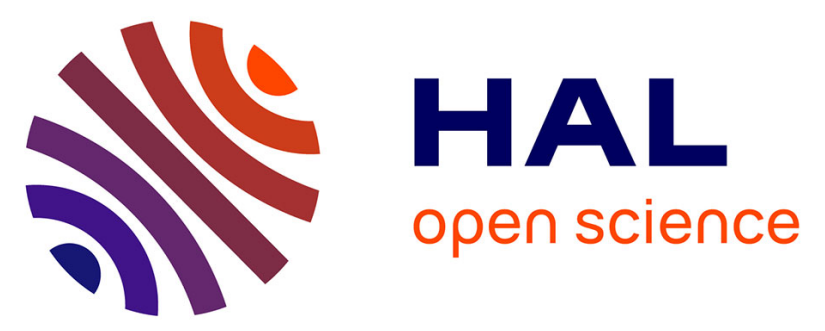

\title{
Effect of a Low Level Imitation Strategy on an Autonomous Multi-Robot System Using On-Line Learning for Cognitive Map Building
}

Abdelhak Chatty, Syed Khursheed Hasnain, Philippe Gaussier, Ilhem Kallel, Philippe Laroque, Adel M. Alimi

\section{To cite this version:}

Abdelhak Chatty, Syed Khursheed Hasnain, Philippe Gaussier, Ilhem Kallel, Philippe Laroque, et al.. Effect of a Low Level Imitation Strategy on an Autonomous Multi-Robot System Using On-Line Learning for Cognitive Map Building. IEEE International Conference on Robotics and Biomimetics (ROBIO 2012), Dec 2012, Guangzhou, China. pp.1-6. hal-00752995

\section{HAL Id: hal-00752995 https://hal.science/hal-00752995}

Submitted on 1 Feb 2013

HAL is a multi-disciplinary open access archive for the deposit and dissemination of scientific research documents, whether they are published or not. The documents may come from teaching and research institutions in France or abroad, or from public or private research centers.
L'archive ouverte pluridisciplinaire HAL, est destinée au dépôt et à la diffusion de documents scientifiques de niveau recherche, publiés ou non, émanant des établissements d'enseignement et de recherche français ou étrangers, des laboratoires publics ou privés. 


\title{
Effect of Low Level Imitation Strategy on an Autonomous Multi-Robot System Using On-Line Learning for Cognitive Map Building.
}

\author{
Abdelhak Chatty ${ }^{1,2}$,Syed Khursheed Hasnain ${ }^{2}$, Philippe Gaussier $^{2}$, Ilhem Kallel ${ }^{1}$,Philipe Laroque ${ }^{2}$, and Adel M. Alimi ${ }^{1}$ \\ ${ }^{1}$ REGIM: REsearch Groups on Intelligent Machine \\ University of Sfax, National School of Engineers (ENIS) Sfax, Tunisia \\ ${ }^{2}$ ETIS: Neuro-cybernetic team, Image and signal Processing \\ National School of Electronics and its Applications (ENSEA), Cergy-Pontoise University, Paris, France \\ \{abdelhak_chatty, ilhem.kallel, adel.alimi\}@ieee.org, \{Gaussier, syed-khursheed.hasnain\}@ensea.fr, laroque@u-cergy.fr
}

\begin{abstract}
In this paper, we present the interest of low level imitation strategy on individual and population levels in the field of Multi-Robot System. Particularly, we show that adding a simple imitation capability to our bio-inspired architecture boosts the ability of individual cognitive map building. Taking into account the notion of imitative behavior, we also show that the individual discoveries in each robot (i.e. goals) could have an effect on population level and therfore it induces a new learning capability at the individual level. To analyze and validate our hypothesis, a series of experiments have been performed with and without a low level imitation strategy.
\end{abstract}

\section{INTRODUCTION}

In an unknown environment, interactions among robots can be based on Stigmergy [1] which reflects indirect communication through the environmental changes. To share partial knowledge of this environment in a cognitive ${ }^{1}$ multi-robot system, which is based on the cognitive map for navigating and planning, several benefits can be expected from imitation capability [2], [3], [4], [5], [6]. The imitation strategy can be considered as a powerful tool for autonomous robots to learn and discover new tasks and places. Learning by imitation and observation is an intuitive and natural method, it is not only the tool for learning but also a way to speed up the learning process. Therefore modern robotics considers imitation as a powerful behavior that enables learning by observation [7] [8]. The idea of imitation learning for robots is inspired by the notion of imitation described by developmental psychologists. According to psychologists immediate or low-level imitation corresponds to the ability of a child (few months old) to imitate (spontaneously) meaningless gestures. This low level imitation may serve to higher level functions for instance, learning by observation [9].

In our case, we associate this concept of very low level imitation with our architecture of exploring environments and creating cognitive maps. In the onset of the experiment, a mobile robot (Robot A) creates its own cognitive map (without

\footnotetext{
${ }^{1}$ Here cognitive means each robot is able to build online an internal cognitive topological map of its environment.
}

the imitation strategy) while exploring the environment to discover the resources for its needs. This random exploration takes a long time to build a cognitive map. As robot A completes its cognitive map, a second mobile robot (Robot B) which is able to perform low level imitation starts exploring the environment. When Robot $\mathrm{A}$ is in the field of view of Robot B, it imitates the first robot by following its current direction of movement. As robot $A$ visits resources several times depending on its needs, robot $\mathrm{B}$ discovers these resources during its low level imitation and builds its own cognitive map. Once the resources are discovered the imitation strategy is turned-off and robot B visits the resources independently depending on its needs.

In section 2 the theory and the bio-inspired architecture of our proposed cognitive map and its adaptive capability are presented. Section 3 describes the imitation process and finally, before concluding, section 4 details experimental results along with comprehensive discussion and analysis on positive feedback of imitation strategy in cognitive multi-robot system.

\section{THE BIO-INSPIRED ARCHITECTURE}

Starting from neurobiological hypothesis which highlights the importance of hippocampus in the spatial navigation, [10], [11], [12], [13] has brought to light special cells in the rat's hippocampus that fire when the animal is at a precise location. These neurons are called place cells (PC). We do not directly use them to navigate, plan or construct a map, we rather use neurons called transition cells (TC) [14]. This kind of cells is inspired by a neurobiological model of temporal sequence's learning in the hippocampus [15], [16], [17]. A transition cell codes a spatio-temporal transition between two PCs successively winning the competition, respectively at time $\mathrm{t}$ and $\delta t$. The set of the PCs and the TCs constitute a noncartesian cognitive map. The reason behind using transition cells is that their association with an action is univocal and quite straightforward. There is no need of an external algorithm to extract the action from the cognitive map. The model [18], also describes the role of the hippocampus: The 
entorhinal cortexwhich is the main input to the hippocampus (EC) which receives signals from associative cortical areas and then filters and merges the multimodal information. In order to transfer it to CA3 pyramidal cells and dentate gyrus (DG), the DG operates an eminence between the signals and puts them together in a temporal hierarchy which later on is retransmitted on CA3 cells. This temporal hierarchy allows CA3 to be aware of past events and put them in correspondence with present events, therefore it behaves like an associative memory through stocking possible transitions between these events. The recognition of the ongoing sequence happens at the level of CA1 using EC and CA3 information. It also extends to the prefrontal cortex (PRC) to serve the higher levels of cognitive processes. Based on the same research, [19] form a model of the cognitive map in the hippocampus representing the entire environment and suggest the shortest paths to a given goal. The model proposed by [20] provides a complete neural architecture of the learning process. His model uses a cognitive map and associates it with a mechanism of action selection. [21], [22] also proposed a model of cognitive embedded in a parieto-fronatl network, which is based on cortical columns.

\section{A. The cognitve map}

To develop a cognitive multi-robot system we took inspiration from the model of [18]. A schematic view of our architecture is shown in figure 1:

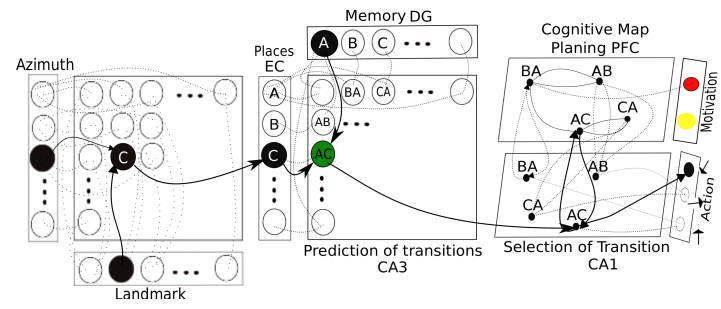

Fig. 1. Model of loop hippocampo-cortical of the creation of the cognitive map

To create the PC, the robot takes a visual panorama of the surrounding environment. A camera mounted on a pan system allows to perceive the environment or the surroundings. The visual images are processed to extract visual landmarks. These landmarks are learnt and a visual code is created by combining the landmarks and their corresponding azimuth. This configuration serves as a code for PCs. The signals provided by the EC are solely spatial and consistent with spatial cells' activities. In order to select (only) the cell with the strongest response at a specific location, spatial cells' activities are submitted to a Winner-Take-All competition. We subsequently speak about the current location by indicating the spatial cell which has the highest activity at a given location. The temporal function at the level of the DG is reduced to the mere memorization the past location. The acquired association at the level of the pyramidal cells CA3 is then the transition from a location to another aside from all information concerning the time spent to carry out this transition. Once the association from the past location and the new one is learned, every new entry will reactivate the corresponding memory in the DG. During the exploration of the environment, the cognitive map is gradually created when the robot moves from one place to another. The equations governing learning in the cognitive map are given below 1 and 2:

$\frac{d W_{i j}^{C C}(t)}{d t}=T(t) \cdot\left(\left(\gamma-W_{i j}^{C C}\right) \cdot X_{i}^{C}(t) \cdot X_{j}^{C}(t)-W_{i j}^{C C}(t) \cdot\left(\lambda 1 \cdot X_{j}^{C}(t)-\lambda 2\right)\right)$

$$
\frac{d W_{i j}^{M C}(t)}{d t}=S(t) \text { fori, } j=\operatorname{argmax}_{k, l}\left(X_{l}^{C}(t) \cdot X_{M}^{K}(t)\right)
$$

$T(t)$ is a binary signal ( 0 or 1$)$ activated when a transition is made (moving from one place to another). This signal controls the learning of recurrent connections $W^{C C} \cdot \gamma$ is a parameter less than 1 which regulates the distribution of motivation activity on the map. $\lambda 1$ and $\lambda 2$ are parameters of active and passive oblivion respectively on the recurrent connections. $S(t)$ is a signal marking the satisfaction of a goal (for example the resources discovery). This signal controls the learning of synaptic connections between neurons in $W^{M C}$ motivations' activity $X^{M}$ and neurons of the cognitive map of activity $X^{C}$. After having explored the environment, the robots are in a position of predicting the locations directly reachable in each situation. Figure 2 presents an example of a cognitive map according to the perception of one robot.

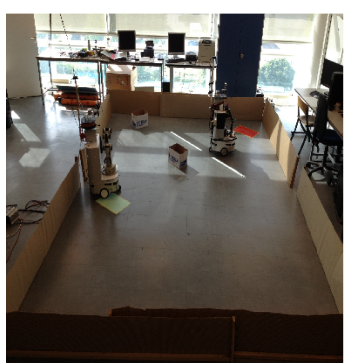

(a)

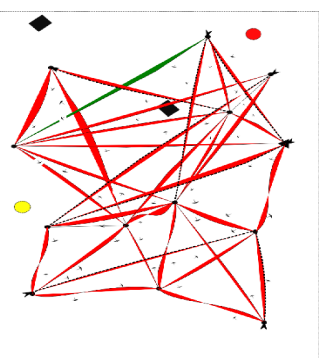

(b)
Fig. 2. From the perception of an unknown environment to the building of a cognitive map. This figure is a simplified view of the the robot's cognitive map based on transition cells. The construction process is based on: place recognition using $\mathrm{PCs}$, activation of one $\mathrm{PC}$ and delayed activation of another allowing for transition prediction (green transition) and the need for sources (R1 and R2) which therefore triggers maximum motivation for a given goal. Figure (a) shows the real environment and figure (b) shows the cognitive map of one robot after 30 minutes of learning.

The environment is composed of 2 sources, 2 obstacles and 2 identical robots (robulab 10 from robosoft) that are able to avoid the stable and dynamic obstacles, to navigate, to learn and to construct their on-line own cognitive map in a unknown environment (with a threshold of vigilance to learn news places equal to 0.65 and a duration of learning equal to 30 minutes). This model has been used in Cognitive multi-robot system navigation tasks [23] where the robots were able to build their cognitive map on-line and learn how to move towards various goals in unknown environment. 


\section{B. The selection of the shortest path}

Our Cognitive robots are also able to select the shortest path [23] in order to reach their targets: when discovering a source (food, nest or water), the motivation associated with it (thirst, hunger or stress) is associated with the cognitive map at the location where it was found. This motivation then spreads to the graph, indicating the shortest path to reach the source from any known location. We can therefore merge these motivation activities with the transition prediction activities coming from the hippocampus. The level of prediction of different transitions from the hippocampus is substantially the same. The activity derived from the cognitive map comes then to predetermine the transition choice among those that have been predicted, through selecting thereby the transition leading the fastest towards the target. A field of neurons gathers the information on the robot's direction during its entire passage of a location. It provides therefore, at the exit of a location, the direction and the length of the trajectory performed at the location. This direction is considered to be the one which is required to perform the transition. During the selection of a particular transition in order to reach the target, we can replicate, in terms of direction, the motion associated which has already been learned during the discovery of the environment.

\section{THE IMITATION PROCESS}

In order to share a partial knowledge of the environment, direct communication between robots could be used. However, the presented architecture is based on the stigmergy to communicate between robots. Thus, imitation seems an intersting way to strengthen this architecture. In this section, we describe a very simple architecture for imitation in a navigation perspective. The proposed model is based on dynamical interactions among mobile robots. Our aim is to provide limited capabilities to a mobile robot to interact dynamically with other robots by following their current direction of movement. Figure 3 shows the architecture of our imitation model.

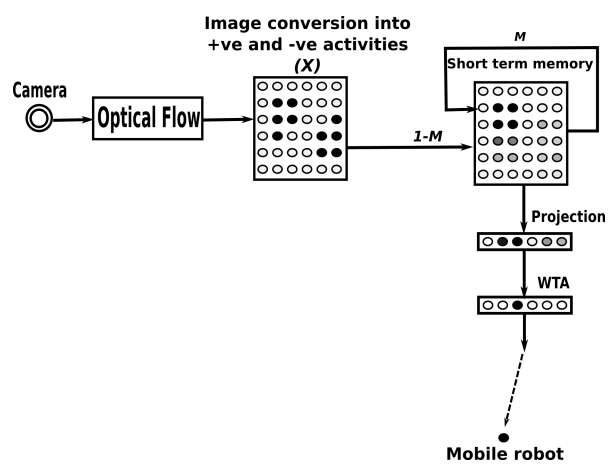

Fig. 3. Architecture to imitate or follow the other robots depending on the estimation of their velocity vectors (optical flow)

As shown on figure 3, perceived motions (by camera) in the visual field (of mobile robot) are estimated by a classical optical flow algorithm [24]. If the perceived motion is in upward direction, it is considered as positive activity, on the other hand, downward motion is accumulated as negative activity. Similarly, motion on the left direction leads to positive activity while movements on the right direction are considered as positive activity. Figure 4 is a snapshot taken during the experiment illustrating optical flow functioning. There are two moving objects in the field of view of a mobile robot. One moves left to right and it is transformed to negative activities by the optical flow (gray and unfilled blocks) while the other moves right to left and transformed into positive activities (shown by black color).

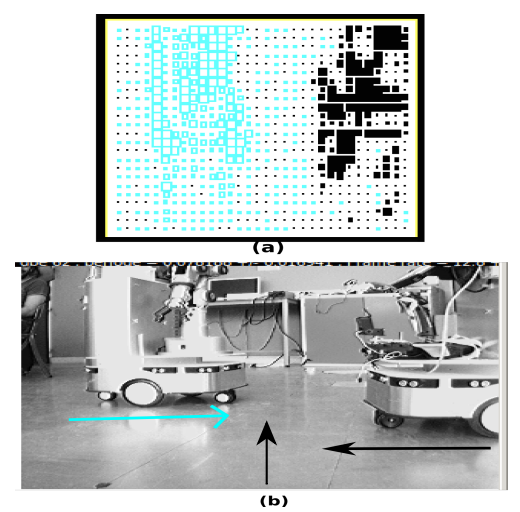

Fig. 4. Optical flow function: left side movements shows as black and realized by $+v e$ activities while right side motion perceived as $-v e$ activities and shown by unfilled blocks. It also shows how an imitator robot perceives the movements of the other robots (the arrows represent the robots directions)

To determine the correct direction (motion) of an interacting partner or to follow the interactor, optical flow activities are transferred to a short term memory block, this block is used to avoid the fast changes in the environment. Then, all the pixels of short term memory module are projected on the $x$-axis (i.e all pixels in each column are added). Than, a Winner Takes All (WTA) selects the highest activated column. This selected column indicates the direction of the movement and the robot can point and follow the interactor. For this experiment the resolution of image is $32 \times 24$ ( 32 columns or location), these 32 possible locations are realized in $60^{\circ}\left(-30^{\circ}\right.$ to $30^{\circ}$ ) circular angles which are fed to the motor according to the corresponding columns (column zero refers to $-30^{0}$ while $32^{\text {th }}$ column corresponds to $30^{\circ}$ ) and $0^{0}$ when the agent stands in front of mobile. If two or multiple visual stimuli are present at the same time then our proposed Imitation algorithm dynamically locates and selects the interacting agent who's estimated velocity vectors are larger.

\section{EXPERIMENTATIONS, RESULTS AND DISCUSSION}

Our experimental results show how imitative strategy can be useful in multi-robot system. We worked on a series of experiments to validate that the imitation strategy, which works among humans to improve the individual and social behaviors, can also be applied on a cognitive multi-robot system.

\section{A. The analysis of the imitation behavior}

In this section, we detail our experimental environment and procedures. To avoid complexities we use minimal setup which 
includes 2 sources, 3 obstacles and 2 robots: leader robot $L R$ (which has already learnt the environment and the positions of both resources R1 and R2) and the imitative cognitive robot $I R$ (which is capable of imitating and of following the other cognitive robot $L R$ and to create its cognitive map on-line). Figure $5 \mathrm{a}, \mathrm{b}, \mathrm{d}$ and e shows that the $I R$ tries to construct its own cognitive map by following the cognitive robot $L R$ (when $L R$ navigates towards the resources using its own cognitive map). Figure $5 \mathrm{c}$ and $\mathrm{f}$ demonstrate that the $I R$ is succeeded in discovering both resources $R 2$ and $R 1$ during the imitation process or following the $L R$. Throughout the experiments, it was notable that the $I R$ also has the capability of learning and of constructing its own cognitive map (on-line) independently, but if the same task is performed by using imitation tactic it boosts the performance (as a function of time) of discovering resources and creating a cognitive map. After discovering the positions of both resources, the IR will be able to return alone to the two resources using its own cognitive map. This experience proves the importance of imitation in multi robot system.

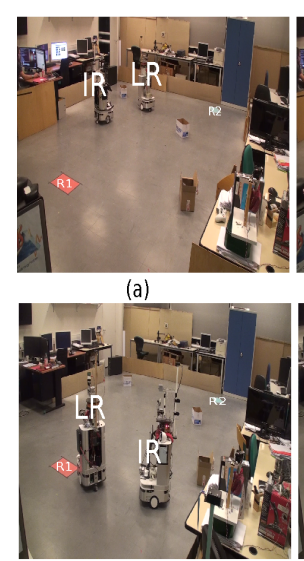

(d)

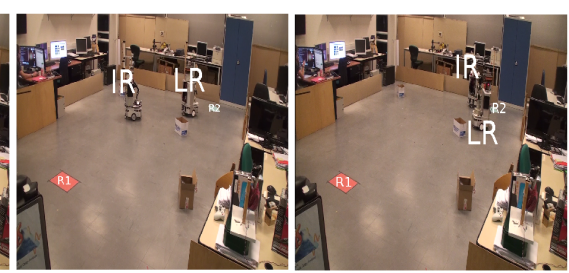

(b)

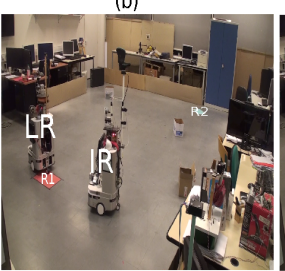

(e)

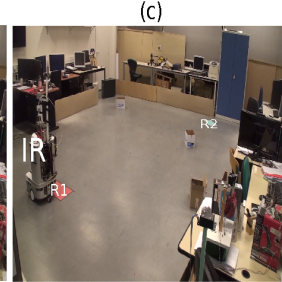

Fig. 5. The influence of the imitation behavior in a multi-robot system. The experiment is done with two robots $I R$ : imitator cognitive robot and a simple cognitive robot $L R$ that has already learned the environment and the position of the resources $\mathrm{R} 1$ and $\mathrm{R} 2$. The figure is presented with chronological order and proves that thanks to imitation, the imitator robot was able to find the two resources R1 and R2. The video is available at http://perso-etis.ensea.fr/neurocyber/Videos/Cognitive_MultiRobot_System /Imitation_LR-IR.mov

Figure 6 demonstrates the trajectories and the individual behavior of robots in more details. The arrows are the initial positions of the two robots. The dashed line sketches the trajectory of $I R$ whereas trajectory of $L R$ is shown by a continuous line. $L R$ 's trajectory shows that the robot tries to go to the position of $L R$ when $I R$ detects it in its visual field. It is evident that when $I R$ is close to the $L R$, its trajectory tries to follow the trajectory of $L R$ (the two trajectories are confounded). The trajectories of the robots show that $I R$ is able to find the two resources R1 and R2 by imitating the trajectory of $L R$. It is worth to notice that instead of following the complete trajectory of $L R, I R$ imitates and follows $L R$ only for $\alpha$ seconds and then stops the imitation strategy in order to detect another robot. In this case it may happen that $I R$ do not detect $L R$ again because $L R$ continue to move and disappear from the visual field of $I R$. Since for this experiment we used only one robot $(L R), I R$ always detects or loses the same robot.

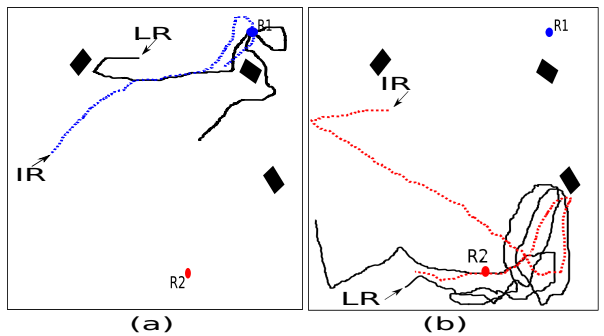

Fig. 6. The trajectories of robots to reach the resources. The cognitive map allows the $L R$ to find the two resources. Thanks to the imitation strategy the $I R$ is able to find the same resources when it follows the trajectory of the $L R$. The $I R$ has the capability to learn the environment and the positions of the resources through creating its own cognitive map. Thus, after the first discovery of the two resources, the $I R$ is able to return alone to the resources. Figure (a) and (b) show the discovery of the resources $R 1$ and $R 2$ respectively.

\section{B. The effect of imitation on the cognitive map}

As the cognitive map is a way to describe the environment, we assumed that the sum of the individual cognitive maps allows to describe all the complexity of the environment. By adding a simple imitation strategy we allow the $I R$ to share this complexity and to learn the experience of the $L R$ to achieve its goals. Since the $I R$ has the capability to create its cognitive map, when it follows the trajectory of the $L R$, its cognitive map will be influenced by the one of the $L R$. Figure $7 \mathrm{a}$ and $\mathrm{b}$ show the cognitive maps of the $L R$ and the $I R$ respectively.

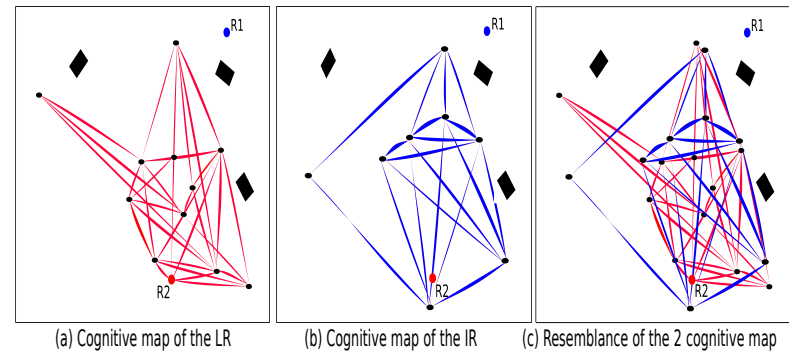

Fig. 7. The influence of the imitation on the creation of the cognitive map of the imitator robot. Figure (a) shows the cognitive map of the $L R$ which already learnt the environment and the positions of both resources R1 and $\mathrm{R} 2$, figure (b) shows the cognitive map of $I R$ that is created after applying the imitation strategy. Figure (c) presents the two cognitive maps in the same plan. The figure proves the positive effect of the imitation strategy which allows the $I R$ to find the two resources and to create a cognitive map which resembles the cognitive map of the $L R$ at the level of the PCs, TCs and the shape of the cognitive map.

In order to see the difference between the two cognitive maps, we put them in the same plan (see figure 7c). It is noticeable that there is a resemblance between the two cognitive maps at the level of the PCs, TCs and the shape of the cognitive maps. Generally, in emergent collective systems, the behavior 
of an agent becomes a cause for the emergent property of another agent and it helps the whole system to solve a specific task. With the imitation strategy we show the same system, In our experiments, $L R$ who had already acquired and learnt the environment, helps another robot to satisfy its motivations. Thus, adding a simple imitation strategy to our cognitive architecture allows the cognitive multi-robot system to help the individual robot.

\section{The effect of imitation on the resources discovery time}

Three different schemes of experiments have been tested to analyze the comparative behavior between a robot having imitative capabilities and a robot without imitation skills during the discovery of the environment and the resources (in our experiments we used 2 resources). For these experiments our main goal is to observe the time of exploration of the resources. For every experiment the robot starts with random positions and resources which are placed randomly. For the first scheme, a single robot moving randomly allows the investigation of the environment and its resources. In the second scheme, the same mobile robot along with a cognitive robot (who already knows the environment and frequently visits the resources according to its needs.) allows the discovery of the resources. The difference between this experiment and the previous one is the way of exploring and the access to resources. In the first experiment, the random robot discovers the resources without any difficulty. However, in the second experiment it is not easy to discover them because the cognitive robot (which has already learned the resources' position) acts as a dynamical obstacle. It means that when both robots are colse to a resource the random robot instead of heading towards this resource it turns in wrong direction to avoid the dynamical obstacle. In this case it loses the learning of the resource's position. Finally, in the last scheme, an imitative robot (that can follow the other robot) along with the cognitive robot allows to discover the resources and the environment. For all these three schemes we interested to compute the discovering time of both resources. For each experiment stated above, we were looking for time to find the resources and every single experiment in each category differs in time (to discover the environment). To find the average time in each category, we conducted several experiments. The number of experiments to take the average value is determined by the Fisher exact test or F test. It is a hypothesis test (statistical), it compares the difference between two variances of two sets of experiments (having the same number of observations) by taking ratio between them;

$$
F=S_{x}^{2} / S_{y}^{2}
$$

where $S_{x}^{2}$ and $S_{y}^{2}$ are the variances of two sets of experiments. $S_{x}^{2}$ is the numerator and it always has the larger value between two variances. If the ratio $(F)$ does not exceed a certain theoretical value (available in the table of fisher), it can be accepted. On the other hand, if $F$ is greater than the theoretical value, we reject the hypothesis of equality of two variances, in this case we increase the number of experiments to close the theoretical value of equality of two variances. For the first category, where a random robot (without imitation) looks for resources, we conducted two sets of experiments (4 experiments in each set) and computed the Fisher value $(F=5.387)$, it is less than 19 which is given in the table of fisher. For the second category, where a random robot explores the environment along with a cognitive robot, we conducted two sets of experiments ( 8 experiment in each set) and computed the Fisher value $(F>100)$, it is greater than 19 and unacceptable. To reduce the difference in variance, we conducted another set of experiment and computed $F=15.79$, it is less than 19 (does not exceed Fisher's theoretical value). Finally, for the last category, where the imitative robot imitates the cognitive robot to find the resources, we applied two sets of experiments (8 experiments in each set) and calculated the Fisher value $(F=2.153)$, it is less than theoretical value (19).

The average time to find two resources for each category is plotted in figure 8. It is clear from the results shown in figure 8 , that the imitative robot takes less time to find both resources ( $\mathrm{R} 1$ and R2), its accumulative time is about 5 minutes ( $2 \mathrm{~min}$. for R1 and $3 \mathrm{~min}$. for R2). However, the random robots of the first and second category take much more time (about 3 times more) to discover resources. The first category's accumulative time is 22 minutes (6 min. for $\mathrm{R} 1$ and $16 \mathrm{~min}$. for $\mathrm{R} 2$ ) similarly, the second takes 19 minutes $(6 \mathrm{~min}$. for R1 and 13 min. for R2).

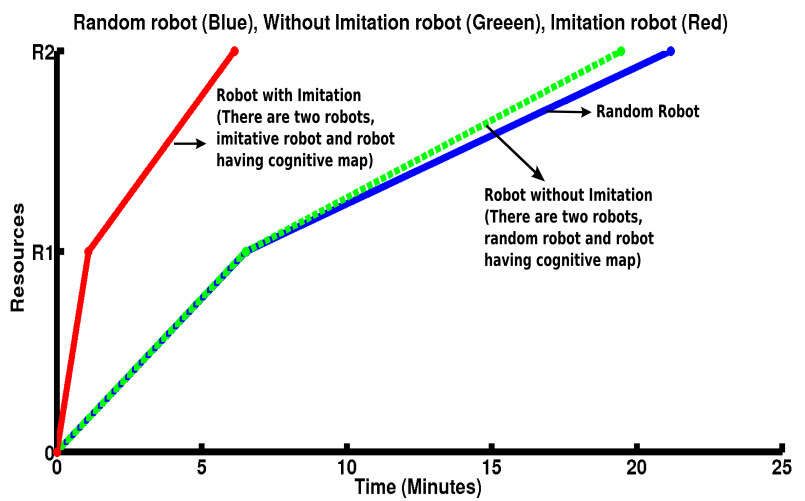

Fig. 8. The average time of the resources discovery. The first curve (from the left) shows the time to discover the resources by a robot having imitation capability along with a cognitive robot which already knows the resources. The second curve (dotted line) describes the time taken by a robot (without imitation capability) to explore the resources along the same cognitive robot as in the previous case. The last curve demonstrates the average time needed to find the resources when single robot explores the environment randomly.

We also have validated the positive feedback of the imitation strategy in a simulated cognitive multi-agent system based on the same bio-inspired architecture. We have shown that with imitation strategy, our cognitive agents are able to optimize the time of discovering the resources as well as increase the average number of the survivors (see figure 9).

By the above stated results, we can conclude that Imitative strategy that is vital in humans for social behaviors can be equally implemented in cognitive multi-robot system for 


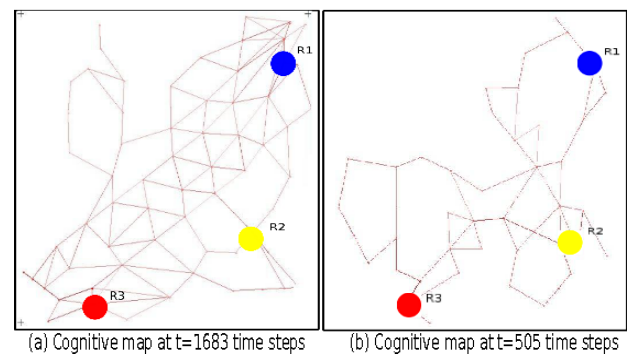

Fig. 9. The positive feedback of the imitation strategy in a simulated cognitive multi-agent system. Figure (a) shows that without imitation cognitive agent_1 is able to find the three resources (R1,R2 and R3) after 1683 time steps (5 minutes). However, in figure (b), the imitation strategy allows cognitive agent_2 to reach the resources after 505 time steps (1,5 minutes).

effective learning and other behaviors. We can also conclude that our bio-inspired architecture is able to maintain the same performances and results whether it is tested by simulated agents or examined by the real robots. Thus, we prove that the cognitive map aims to teach our cognitive robots the way to navigate, to reach the sources, avoid planning problems before complete exploration of the environment and adapt to a dynamic environment. Moreover, adding a simple imitation strategy to our bio-inspired architecture, allows our cognitive robots to have the same behavior as the ants algorithm (when they try to look for food) but without the need to leave physical trace in the environment.

\section{CONCLUSiON}

In this paper, we highlighted the importance of the imitation strategy. It boosts the capability (as a function of time) of a cognitive multi-robot system to adapt to an unknown environment. It allows to solve the navigation task among various targets. We proved that combining the learning capability with simple imitation tactic leads (in a real multi-robot system) to a positive feedback in the individual and population level. Moreover, it optimizes the time to explore the resources, and it allows to create a cognitive map for the imitator robot $I R$ (approximately the same shape as the Leader robot $L R$ ). Thus, to keep the rules of the stigmergy, imitation strategy is a better way to share the knowledge between robots without direct communication between them. As prospects, we are trying to study the effect of the imitation in cognitive multi-robot system which is able to take and withdraw objects.

\section{ACKNOWLEDGMENT}

The authors would like to thank the financial support of the Tunisian General Direction of Scientific Research and Technological Renovation (DGRSRT), under the ARUB program 01/UR/11 02, the Institut Français de Tunisie, the INTERACT french project referenced ANR_09_CORD_014, the NEUROBOT french project referenced ANR-BLAN-SIMI2-L2-100617-13-01 and DIGITEO project AUTO EVAL.

\section{REFERENCES}

[1] O. Holland and C. Melhuish, "Stigmergy, self-organization, and sorting in collective robotics," Artif. Life, vol. 5, no. 2, pp. 173-202, 1999
[2] P. Gaussier, S. Moga, J. P. Banquet, M. Quoy, and N. E. Modelisations, "From perception-action loops to imitation processes: A bottom-up approach of learning by imitation," 1997.

[3] P. Laroque, N. Gaussier, N. Cuperlier, M. Quoy, and P. Gaussier, "Cognitive map plasticity and imitation strategies to improve individual and social behaviors of autonomous agents," Journal of Behavioral Robotics, 2010.

[4] S. Schaal, J. Peters, J. Nakanishi, and A. Ijspeert, "control, planning, learning, and imitation with dynamic movement primitives," in workshop on bilateral paradigms on humans and humanoids, ieee international conference on intelligent robots and systems (iros 2003), 2003.

[5] T. Chaminade, E. Oztop, G. Cheng, and M. Kawato, "From selfobservation to imitation: visuomotor association on a robotic hand." Brain research bulletin, vol. 75, no. 6, pp. 775-784, Apr. 2008.

[6] M. Lagarde, P. Andry, P. Gaussier, S. Boucenna, and L. Hafemeister, "Proprioception and Imitation: On the Road to Agent Individuation," in From Motor Learning to Interaction Learning in Robots, O. Sigaud and J. Peters, Eds. Berlin, Heidelberg: Springer Berlin Heidelberg, 2010, vol. 264, book part (with own title) 3, pp. 43-63.

[7] Y. Kuniyoshi, N. Kita, S. Rougeaux, S. Sakane, M. Ishii, and M. Kakikura, "Cooperation by observation - the framework and basic task patterns," in ICRA, 1994, pp. 767-774.

[8] S. Schaal, "is imitation learning the route to humanoid robots?" no. 6 , pp. 233-242, 1999.

[9] A. de Rengervé, S. Boucenna, P. Andry, and P. Gaussier, "Emergent Imitative Behavior on a Robotic Arm Based on Visuo-Motor Associative Memories," in IEEE/RSJ International Conference on Intelligent Robots and systems (IROS'10), Taipei, Taiwan, October 2010, pp. 1754-1759.

[10] J. O'Keefe and L. Nadel, The hippocampus as a cognitive map / John O'Keefe and Lynn Nadel. Clarendon Press ; Oxford University Press, Oxford, 1978.

[11] N. Burgess, J. G. Donnett, and J. O’Keefe, "Robotic and neuronal simulation of hippocampal navigation," University of Manchester, vol. 352, pp. 1361-6161, 1997.

[12] M. Milford and G. Wyeth, "Mapping a suburb with a single camera using a biologically inspired slam system," IEEE Transactions on Robotics, vol. 24, no. 5, pp. 1038-1053, October 2008.

[13] I. A. Bachelder and A. M. Waxman, "Mobile robot visual mapping and localization: A view-based neurocomputational architecture that emulates hippocampal place learning," Neural Networks, vol. 7, pp. 1083 - 1099, 1994.

[14] P. Gaussier, A. Revel, J. P. Banquet, and V. Babeau, "From view cells and place cells to cognitive map learning: processing stages of the hippocampal system." Biological Cybernetics, vol. 86, no. 1, pp. 15$28,2002$.

[15] A. Alvernhe, T. Van Cauter, E. Save, and B. Poucet, "Different CA1 and CA3 representations of novel routes in a shortcut situation." The Journal of neuroscience : the official journal of the Society for Neuroscience, vol. 28, no. 29, pp. 7324-7333, Jul. 2008.

[16] E. V. Lubenov and A. G. Siapas, "Hippocampal theta oscillations are travelling waves," Nature, vol. 459, no. 7246, pp. 534-539, May 2009.

[17] D. M. Smith and S. J. Y. Mizumori, "Hippocampal place cells, context, and episodic memory," Hippocampus, pp. 716-729, 2006.

[18] J. P. Banquet, P. Gaussier, J. C. Dreher, C. Joulain, A. Revel, and W. Gunther, "Spacetime, order and hierarchy in fronto-hippocamal system : A neural basis of personality. in cognitive science perspectives on personality and emotion." Elsevier Science BV, p. 123189, 1997.

[19] R. U. Muller, M. Stead, and J. Pach, "The hippocampus as a cognitive graph," 1996.

[20] M. E. Hasselmo and H. Eichenbaum, "Hippocampal mechanisms for the context-dependent retrieval of episodes." Neural Netw, vol. 18, no. 9, pp. 1172-1190, Nov. 2005.

[21] L.-E. Martinet, D. Sheynikhovich, K. Benchenane, and A. Arleo, "Spatial Learning and Action Planning in a Prefrontal Cortical Network Model," PLoS Comput Biol, vol. 7, no. 5, May 2011.

[22] M. A. Arbib, "Autonomous robots based on inspiration from biology: The relation to neuroinformatics," Neuroinformatics, vol. 3, no. 3, pp. 281-286, 2005.

[23] A.Chatty, P.Gaussier, I.Kallel, P.Laroque, and A.M.Alimi, "Adaptation capability of cognitive map improves behaviors of social robots," in IEEE Conference on Development and Learning and the Epigenetic Robotics, Juillet. 2012.

[24] B. K. P. Horn and B. G. Schunck, "Determining optical flow," Artif. Intell., vol. 17, no. 1-3, pp. 185-203, 1981. 